\title{
Numerical Simulations of the Cavitating Flow on a Marine Propeller
}

\author{
NaiXian Lu \\ Chalmers University of Technology \\ Gothenburg, Sweden \\ Göran Bark \\ Chalmers University of Technology \\ Gothenburg, Sweden
}

\author{
Urban Svennberg \\ Rolls Royce AB \\ Kristinehamn, Sweden \\ Rickard Bensow \\ Chalmers University of Technology \\ Gothenburg, Sweden
}

\begin{abstract}
This paper deals with numerical simulations of the cavitating flow around a marine propeller operating in open water but mounted on an inclined shaft. The investigation is mainly based on both Large Eddy simulation (LES) and Unsteady Reynolds-Averaged Navier-Stokes (URANS) in combination with a Volume-of-Fluid implementation to capture the liquid-vapour interface and a transport equation-based method for the mass transfer between the phases. Potential flow solver result will also be included to offer a complete picture of the general behaviour and capabilities of a range of computational methods with different levels of detail. Highspeed video recordings from experiments are available for detailed inspection.
\end{abstract}

\section{INTRODUCTION}

Propeller cavitation is of major concern for vessels in terms of performance degradation, erosion and passenger comfort due to cavitation induced vibrations and noise. However, with increasing demand for faster vessels, and at the same time, for higher propulsive efficiency, it is favourable to decrease the margin of cavitation-free operation or to allow for some "controlled" amounts of sheet cavitation on the propeller blades. These two contradictory demands cause the propeller design to be a game of balancing the pros and cons, and it becomes crucial to be able to determine the characteristics of cavitation and not only its appearance or extent. A common way to study propeller cavitation is through model scale experiments. However, this method has several drawbacks, such as high cost, long execution time, scaling effects, and perhaps more importantly, the limited measurable data that can only indicate the possible existence of a certain problem and will give more limited guidance regarding how to redesign. All these constraints have cultivated the need for developing a reliable, versatile and robust computational tool, to better understand the phenomenon itself as well as to contribute in advanced prediction and design work.

Today the standard design tools typically include potential flow solvers on the basis of lifting-line/lifting surface theory, able to predict a reasonable pressure distribution on the blade, but with clear theoretical limitations regarding cavitation dynamics. The use of RANS, Reynolds-Averaged NavierStokes, method has become more feasible as computational resources become more affordable. However, although some general features such as cavity extent or shedding behaviour are to a large extent predictable, the capacity to capture and analyse the detailed dynamics that are responsible for noise and erosion problem is somewhat questionable due to the statistical character of RANS in its way of modelling turbulence. This has motivated the study of using a higher-end yet more costly approach: LES, Large Eddy Simulation, which has the ability to treat highly unsteady processes in a physically realistic manner by resolving large energy-containing scales and modelling the more homogenous energy-dissipating scales.

The propeller chosen for this study is a prototype that is typical for yachts and Ro-Pax vessels and the problem underlying this study is described in [1]. When designing propellers for a passenger ship, human comfort is of great importance and therefore vibration and noise is crucial when evaluating the performance of the propeller. Tip vortex cavitation, being one of the main sources of noise when the propeller is operating, is sensitive to manipulation by modification of the blade tip geometry to delay the onset and character of its occurrence. Therefore, experiments have been conducted at RRHRC, the Hydrodynamics Research Centre of Rolls-Royce AB, Kristinehamn, Sweden, to study tip vortex strength and related noise of a series of propellers.

In this work the cavitating flow around a marine propeller is simulated with three computational methods, ranging from lifting surface methods and RANS to LES, to demonstrate the capability of different simulation tools for this complex flow case where not only performance degradation and cavitation extent suffices to assess the propeller performance. The modeltested propeller was mounted upstream of the shaft that was tilted 10 degrees to obtain a periodically varying blade load and consequently an unsteady behaviour of the cavity, but still in a very well defined inflow suitable for comparisons with computations. The test was carried out in the free surface cavitation tunnel T31 at RRHRC, but the water surface was blocked in the test section by a plate to eliminate the free water surface effects. The numerical simulations are set up with as exact set-up of the experiment as reasonably possible. 
The performances of different solvers in simulating cavitating propeller flow have been summarized in a recent workshop on cavitation and propeller performance [2], supporting the above discussion on merits of potential flow solvers, RANS, and LES. Thus, RANS is expected to be able to predict reasonably accurately cavity extent, but regarding the cavitation dynamics, results presented at [2] indicate that scale resolving methods, like DES or LES, are needed. However, there are reports where also RANS display some reasonable dynamic features, see e.g. [4]. LES results have proven promising, [3], [5] but has not been tested for a modern propeller design and its complicated cavitation problems. One objective of the study presented in this paper is thus to test the merits of available computational tools for this flow.

The structure of the paper is as follows: Firstly the computational models are briefly stated and the computational setups are given. Experimental observations are then summarized, followed by the detailed analysis of simulated results. Finally the findings are summarized and suggestions for further improvements are proposed.

\section{SIMULATING CAVITATING FLOWS}

The propeller flow case described above is implemented and simulated using potential flow, RANS and LES solvers. Both LES and RANS simulate the cavitating flow in combination with a single fluid mixture assumption based on a Volume-of-Fluid implementation [6] to capture the liquidvapour interface based on the vapour volume fraction $\alpha_{\mathrm{v}}$. The transport equation-based method based on the work of Kunz et al. [7] and Sauer et al. [8] is employed for the LES and RANS simulation respectively to take account for the mass transfer between the phases. The flow is treated as incompressible. The LES simulation is carried out using an implicit LES approach, implemented using the OpenFOAM libraries, where the numerical dissipation mimic the subgrid effects, this methodology is discussed in details in [3] and the entire modelling technique is presented in [9]. The transient RANS simulation is conducted with k-omega SST model [10] using ANSYS Fluent 13 and the potential solver MPUF-3A is based on vortex-lattice method [11].

\section{COMPUTATIONAL CONFIGURATIONS}

In the experiments carried out at RRHRC, the propeller is mounted upstream of the shaft that is tilted 10 degrees, this imposes a periodically varying load to the blade and consequently obtains an unsteady behaviour of the cavity. The diameter of the model is $0.254 \mathrm{~m}$ with a pitch ratio $\mathrm{P} / \mathrm{D}$ of 1.447 , advance velocity is $4.2 \mathrm{~m} / \mathrm{s}$ and the rotational speed is $17.7 \mathrm{rev} / \mathrm{s}$, resulting in an advanced coefficient $\mathrm{J}=0.934$. This condition imposes much higher load on the propeller blade than it was originally designed in order to provoke inception of cavitation in the experiment.

MPUF-3A takes directly this information as input, together with a wake file that defines the inclined inflow on to the propeller resulting from the tilted shaft. The propeller geometry is specified by a set of non-dimensional radii $r / R$ at which all propeller geometrical quantities such as pitch ratio, rake ratio, skew angle and chord ratio, are supplied. The discretisation of the propeller blade is in accordance with the recommended settings for reasonable accurate and quick results [1].

The computational grids for the viscous calculation are composed of tetrahedrals with prism layer of hexahedrals around the blade, hub and shaft, Fig. 1(d). The structured part is created by Gridgen and the unstructured part is done by Tgrid. In the RANS computation, the fluid domain is divided to a stationary and rotating zone, see Fig. 1(c) and the sliding mesh method is applied. The rotating domain is composed of 6.6 millions cells and the stationary of 2.2 millions. The near wall resolution of the blade is around $\mathrm{y}^{+}=70-100$ on the major part of the blades with maximum levels of 130 locally at the edges of the blades and as low as $\mathrm{y}^{+}=3$ close to the root of the blades. The RANS grid tries to mimic the experiment as much as possible by including the real geometry of the shaft and the actual size of the cavitation tunnel, see Fig. 1(b).

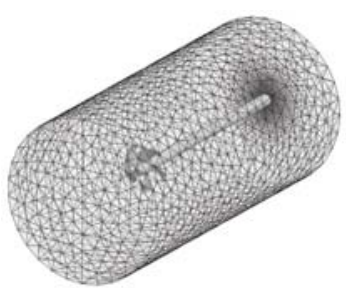

(a)

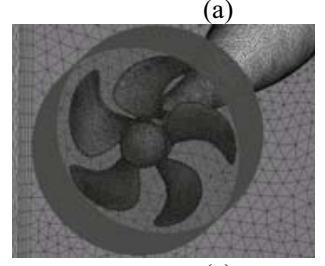

(c)

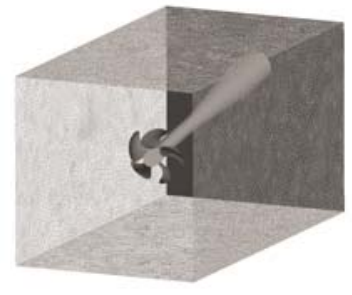

(b)

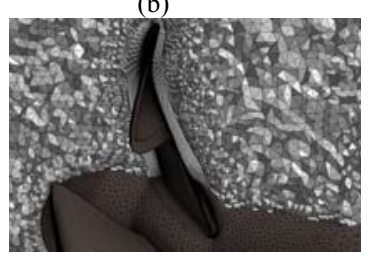

(d)
Figure 1: Computational domain for LES (a) and RANS (b), the rotating zone of RANS computation (c), and details of the mesh (d).

In LES, in order to avoid the need for relative motion between the propeller and the external domain, the outer domain is simplified to a cylinder rotating together with the propeller, Fig. 1(a). The propeller shaft is kept horizontal in order avoid numerical instabilities on the cylindrical boundary and instead the inclined inflow to the propeller is created by translating the domain upwards at $0.729 \mathrm{~m} / \mathrm{s}$. We remark that the LES computation uses the same mesh as the RANS computation close to the propeller, the rotating zone in Fig. $1(\mathrm{c})$, and the total mesh size is 7.8 million cells. For a LES computation, this wall resolution is not sufficient enough to capture correctly the strength of internal jets, [9], therefore the LES computation in this study is expected to bear some defects and need for improvement. Simulations on a grid with finer wall resolution have been started but are not finalized.

Both RANS and LES computations are started without the cavitation source activated to let the wetted flow develop and achieve a stabilized pressure field. Thereafter, the cavitation source is gradually increased from zero to full value over a number of time steps to avoid numerical problems. 


\section{EXPERIMENTAL OBSERVATIONS}

Before going into detailed description, it is vital to point out that the behaviour of the cavitation exhibited in the experiment is rather intermittent, mainly due to the fact that the propeller model is very sensitive to the pitch setting. A deviation of 0.01 degrees between blades can result in substantial difference in the amount of cavitation occurring on blade, illustrated by Fig. 2, Furthermore, the extent of cavitation in the experiments were sensitive to the amount of gas in the water, making quantitative comparison with the numerical simulation is therefore difficult. However here we focus on investigating the physical mechanisms that control the development of cavitation, and they are essential to be captured in a qualified numerical simulation.

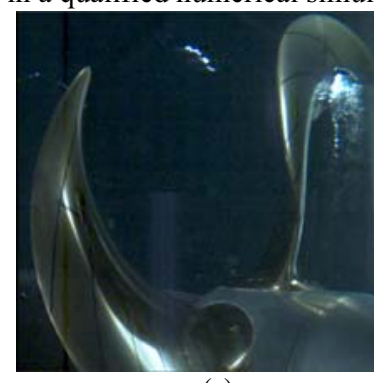

(a)

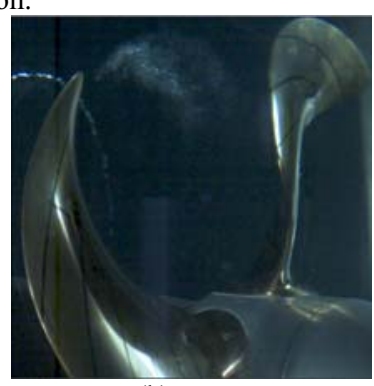

(b)
Figure 2: Two different blades pass by the same location exerting very different cavitation behaviour: The left frame indicating a travelling sheet of mainly bubbles occurred around $r / R \approx 0.8$ in the downstream region of the blade, whereas the right frame indicates no cavitation at this instant.

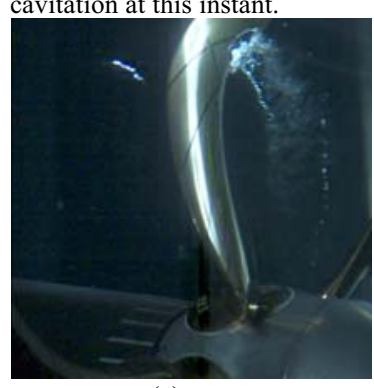

(a)

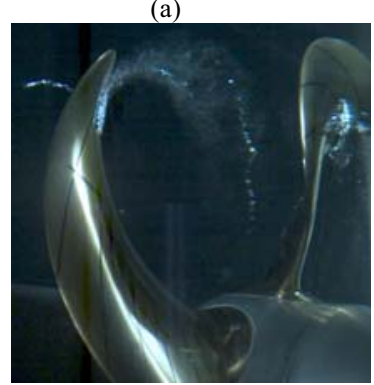

(c)

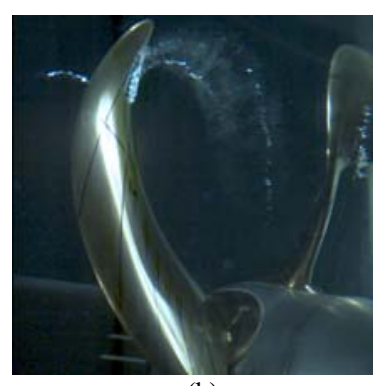

(b)

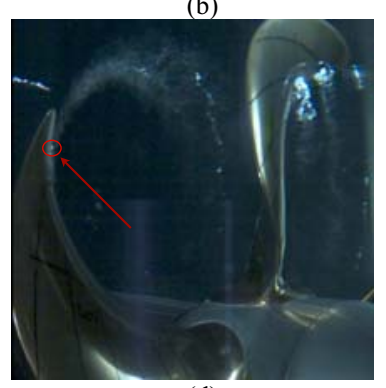

(d)
Figure 3: Development of the sheet around $\mathrm{r} / \mathrm{R} \approx 0.8$ through one blade passage. Frame (a) indicates a travelling bubbly sheet that starts to collapse from its downstream end, at least two vortices are seen. These leading edge vortices at the downstream end of the sheet control much of collapse behaviour, and possibly the interaction between the cavitation and the vortex has moved the final collapse point in frame (d) outwards towards the blade tip. The video is recorded at $20000 \mathrm{f} / \mathrm{s}$.
Figure 3 illustrates a typical cycle of cavitation development through one blade passage. A travelling sheet of mainly bubbles is observed around $\mathrm{r} / \mathrm{R} \approx 0.8$ in the downstream region of the blade. This sheet suffers possibly from a low Reynolds number effect due to the lack of surface roughness in the test, and from the experiment footage it typically starts as a thick and short glassy sheet, which after a short distance transfers into a bubbly sheet - the structure that is expected at full scale, and also in model scale with surface roughness applied. The glassy upstream part of this sheet seems to be controlled by a laminar separation, followed by a transition to turbulence and bubbly structure. This sheet is enhanced by bubbles from occasionally appearing attached sheets upstream the bubble sheet - a typical behaviour for propeller cavitation. This type of cavitation can often be erosive and in the present case scattered collapses were of the erosive type, although rather weak. The downstream end of this sheet is captured by a trailing edge vortex that controls much of the collapse behaviour. During the collapse of the sheet, Fig. 3(a) to (c), traces of at least one vortical structure are visualized by the cavitation. In Fig. 3(d) the sheet has completely disappeared and the final collapse point has climbed towards the blade tip, possibly due to the interaction between the vortex and sheet. At the same instant two visible vortices are taking control of the development of the sheet on the next blade. It is therefore of fundamental interest to understand how these vortices control the growth and collapse of the sheet, and to provide guidelines and vital information for control of this cavitation.

Root cavitation that is partly composed of large travelling single bubbles and partly of glassy sheet is also present; however no dedicated filming was made. A thin tip vortex cavity starting far out on or downstream the blade tip is sporadically observed as well.

\section{SIMULATION RESULTS}

\section{Noncavitating Flow}

The values of thrust coefficient $\mathrm{K}_{\mathrm{T}}$ and torque coefficient $\mathrm{K}_{\mathrm{Q}}$ for the fully wetted condition are listed in Table 1. Blades as well as the hub are included in the listed values. The values obtained by LES are time averaged over about 1.5 revolutions, whereas for the RANS simulation was run for 10 revolutions to establish the flow field and the forces and moments are averaged over the $11^{\text {th }}$ revolution. MPUF is in general performing a satisfying job although it over-predicts the $\mathrm{K}_{\mathrm{Q}}$ value by $4.2 \%$. The over-prediction of LES computation seems to be related to insufficient wall resolution. When $\mathrm{y}^{+}$is decreased from 100 to 10 , the result is improved from $13.7 \%$ to $5.1 \%$ over-prediction for $\mathrm{K}_{\mathrm{T}}$ and $12.7 \%$ to $1.5 \%$ for $\mathrm{K}_{\mathrm{Q}}$. The finer wall resolution computation is only included here to see the trend of the global quantities with increased resolution. The following detailed comparisons of the cavitating flow are still performed on the coarser mesh. RANS performs a moderate job between MPUF and LES with an under-prediction of $9.2 \%$ in $\mathrm{K}_{\mathrm{T}}$ and $2.4 \%$ in $\mathrm{K}_{\mathrm{Q}}$.

The pressure distribution predicted by RANS and LES are shown in Fig. 4, with the second invariant of the vorticity as a greyish iso-surface, indicating vortical flow structures. The tip 
vortex can be seen for both methods and in the LES prediction there is a vortical structure seen on the blade.

\begin{tabular}{c|lcc}
\multicolumn{4}{c}{ Table 1: Open water coefficients in noncavitating conditions } \\
\hline \hline \multirow{5}{*}{ Wetted } & \multicolumn{1}{c}{$\mathrm{K}_{\mathrm{T}}$} & $10 \mathrm{~K}_{\mathrm{Q}}$ \\
\hline \hline \multirow{5}{*}{ Flow } & Experiment & 0.292 & 0.620 \\
& MPUF & 0.291 & 0.646 \\
& RANS & 0.265 & 0.605 \\
& LES $\left(\mathrm{y}^{+}=100\right)$ & 0.332 & 0.699 \\
& LES $\left(\mathrm{y}^{+}=10\right)$ & 0.307 & 0.629 \\
\hline \hline
\end{tabular}

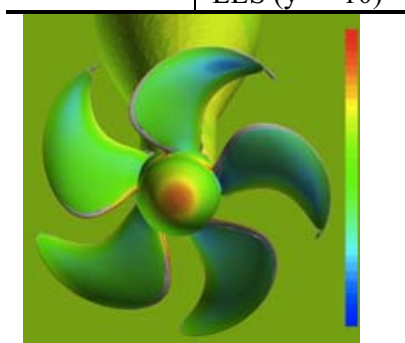

(a)

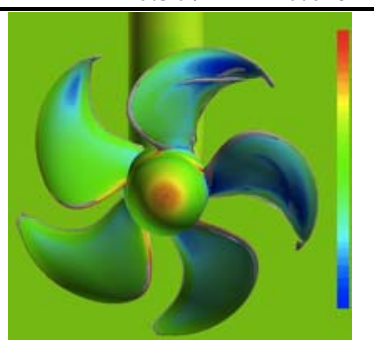

(b)
Figure 4: Blade pressure with iso-surface of the second invariant of the vorticity $\|\nabla \mathbf{v}\|-\|\nabla \times \mathbf{v}\|$, indicating vortical structures, as predicted by RANS (a) and LES (b).

\section{Cavitating Flow}

For the cavitating condition, MPUF performs the best in $\mathrm{K}_{\mathrm{T}}$ with a $0.3 \%$ over-prediction, followed by RANS with $9.0 \%$ under-prediction and finally LES an over-prediction of 9.3\%. For $\mathrm{K}_{\mathrm{Q}}$ RANS is the closest to the experimental value with a $2.1 \%$ under-prediction, followed by MPUF with $4.2 \%$ overprediction and finally LES an over-prediction of $8.4 \%$. However the vulnerability in LES in these global quantities does not represent the whole picture of its ability, and the advantage of MPUF shown in Table 1 and 2 has completely disappeared when it comes to the existence and physics of cavitation, making the reliability of the prediction questionable.

\begin{tabular}{c|lcc}
\multicolumn{5}{l}{ Table 2: Open water coefficients in cavitating conditions } \\
\hline \hline & & $\mathrm{K}_{\mathrm{T}}$ & $10 \mathrm{~K}_{\mathrm{Q}}$ \\
\hline \hline \multirow{3}{*}{ Cavitating } & Experiment & 0.289 & 0.618 \\
Flow & MPUF & 0.290 & 0.644 \\
& RANS & 0.263 & 0.605 \\
& LES $\left(\mathrm{y}^{+}=100\right)$ & 0.316 & 0.670 \\
\hline
\end{tabular}

Figure 5 illustrates the comparison between experiment, left column, and the LES simulation, right column, for the corresponding state in the cavity development. In the simulation visualizations, the cavity interface is indicated by the iso-surface of the vapour volume fraction $\alpha_{v}=0.5$. The first observation is that comparing with the experiment, the simulated cavity extent is larger, however the shed cavities do not survive as long as they do in reality. The former difference can partly be explained by the lack of surface roughness in the experiment, while the latter one is most likely due to the combined effect of a low grid resolution at the blade trailing edge and the chosen value of vapour volume fraction. For tracing cloud cavitation towards the collapse lower $\alpha_{\mathrm{v}}$ would be considered.
Despite the defects mentioned above, the simulation does capture some important flow features; the most important being the vortical structure(s) developed on blade that dominate the growth and affect the collapse of the cavity. Examining the experimental frames, two main mechanisms exhibited are as follows:

(i) The growth of the sheet from the vortex core and the spread towards the blade tip;

(ii) The upstream moving collapse of the main sheet and the shedding of small cavities approximately along the constant radius line.

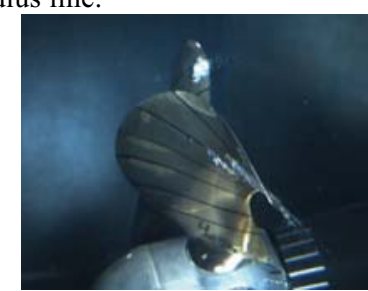

(a)

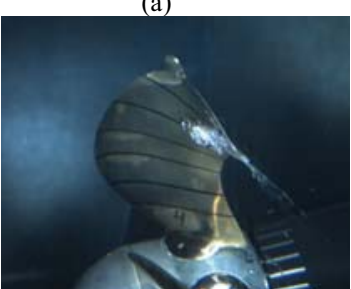

(c)
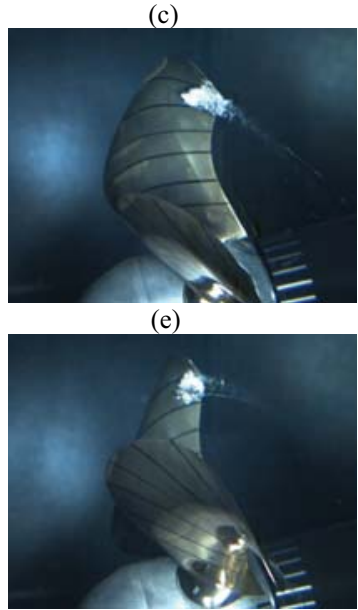

(g)

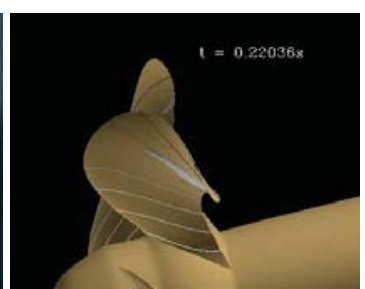

(b)

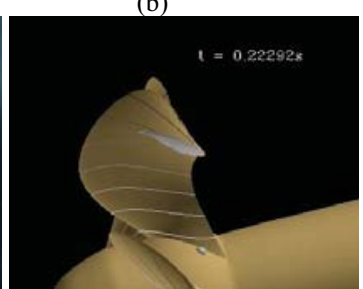

(d)

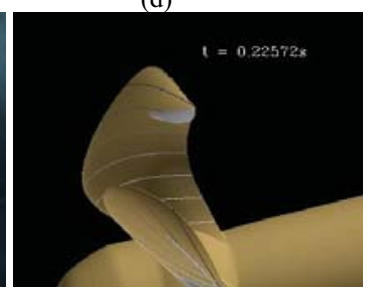

(f)

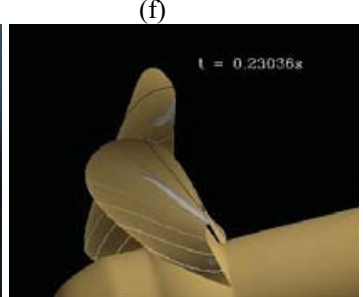

(h)
Figure 5: Development of the cavity during one blade passage. The left column shows the experimental sample frames from a high-speed video recorded at $20000 \mathrm{fps}$. The right column illustrates the LES results of simulated cavity interface defined by vapour volume fraction $\alpha_{v}=0.5$

The simulated dynamics, shown in the right column of Fig. 5 display the same behaviour as the experiment. In Fig. 5(b), the cavity has already grown for some time in the core of an on-blade vortex along the $80 \%$ constant radius line. The illustration of this vortex core will be presented in the following discussion. The location of this sheet cavity coincides very well with the experimental frame Fig. 5(a). In the next frame Fig. 
5(c) the frontal end of the sheet has moved downstream the mid-chord position. In the simulation this change is reflected by a shrinkage of the stream-wise sheet length, Fig. 5(d). Some root cavitation as well is visible in this simulation frame, corresponding to the experimentally reported root cavitation composed of large travelling single bubbles. Moving on to the following state, in the experiment the sheet is clearly making an upstream moving collapse, Fig. 5(e) and (g) where the trailing edge of the sheet is moving in the upstream direction leaving a group of cavitating vortices behind. Traces of at least two vortical structures can be seen by the shed cavities in Fig. 5(g). These flow details are not captured in the simulation. Demonstrated by the previous studies on hydrofoils [9], the spatial requirement is proven to be much higher than the one used in the present study. The shape of the disappearing cavity however to some extent demonstrates the orientation of the vortex that is still in good agreement with the experiment, Fig. 5(f). A slight difference of the location of the remained cavity is also noticed, while the simulated cavity is still located at $\mathrm{r} / \mathrm{R}$ $\approx 0.8$ and the experimental one has moved towards the blade tip and eventually the final collapse point is around $r / R \approx 0.9$. One major difference between Fig. $5(\mathrm{~g})$ and $(\mathrm{h})$ is that in the experiment the blade to be examined subsequently exhibits no occurrence of cavitation, while in the simulation the behaviour is periodic with blade passages. This intermittency demonstrates a non-homogenous nuclei distribution at the model test.

The simulated results by RANS exhibit the existence of a leading edge sheet which starts its development at the most heavily loaded blade position, Fig. 6(a), grows in Fig. 6(c) and then disappears Fig. 6(e),(g), as the load changes on the blade. This leading edge sheet is neither present in the experimental footage nor in the LES simulation illustrated by the right column of Fig. 6, each of which showing the same blade position as in the left column. The LES predicted pressure distribution on the suction side of the blade is globally lower than the prediction of RANS, which explains the large variation in $\mathrm{K}_{\mathrm{T}}$ obtained by the two methods.

Except for the difference in the occurrence of leading edge sheet and pressure level, another major distinction is the shape of the sheet close to the blade tip. This sheet in the LES prediction grows from a vortex core shown in Fig. 7(a) on blade one. Close investigation reveals that there are two vortical structures developed and the cavity grows from the slightly stronger vortex; the other vortex gradually dies out as the sheet grows, in Fig. 7(b), and the stronger vortex is seen to have been enhanced with the disappearance of the sheet, see blade two in Fig. 7(b) compared with blade one. The existence of these vortical structures is supported by the experimental images, Fig. 8. The creation of this vortex is however subject to further studies. The cavities start as thick and short glassy sheets, Fig. 8(a), that transfer to bubbly sheets, Fig. 8(b), where two main vortical structures are visible and possibly a third one exists close to the blade tip. In the next frame Fig. 8(c) the remaining two sheets are collapsing from both ends, with the downstream end moving faster towards the final collapsing point. The rotational motion of the shed small cavitation bubbles indicates the presence of the vortices which are in much control of the collapse. With refined resolution in the region of interest, LES should be able to capture these smallscale effects and offer a better understanding on the cavityvortex interaction. The RANS result indicates no obvious such vortical structures and the shrinking of the sheet is very much related to the variation of pressure and has a more symmetric behaviour.

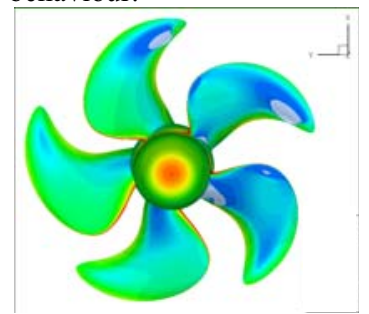

(a)

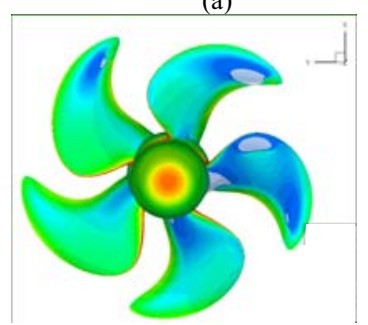

(c)

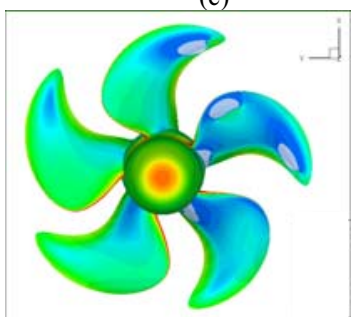

(e)

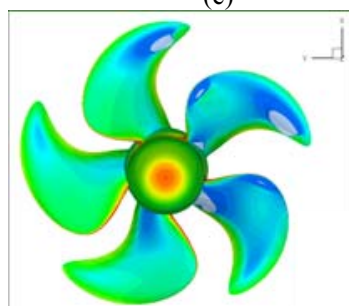

(g)

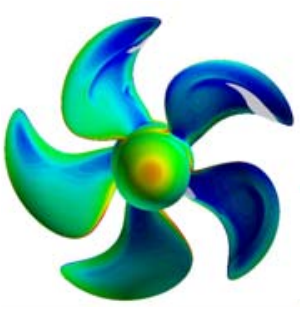

(b)

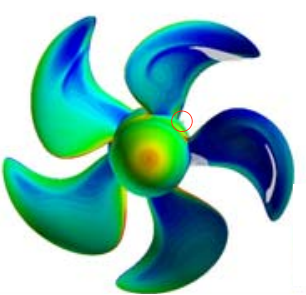

(d)

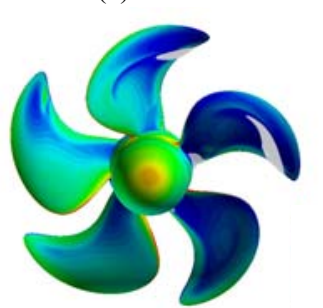

(f)

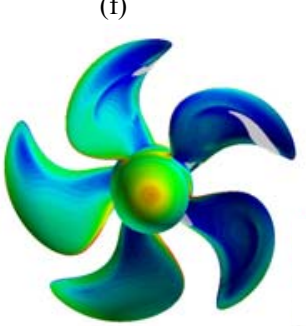

(h)
Figure 6: Pressure distribution on blades and cavity interface defined by $\alpha_{v}=0.5$ as predicted by RANS (left) and LES (right).

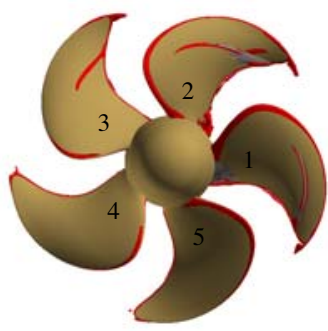

(a)

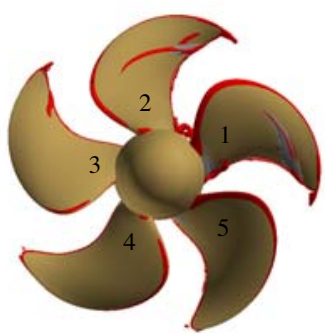

(b)
Figure 7:LES predicted iso-surface of the second invariant of the vorticity $\|\nabla \mathbf{v}\|-\|\nabla \times \mathbf{v}\|$ at selected value (red) and $\alpha_{v}=0.5$ (grey). 
Another interesting finding from the LES result is that on blade the vortex is controlling the behaviour of the collapse, whereas close to the root region, illustrated by Fig. 7(b) it is the other way around that the collapse motion has induced a vortical structure which results in a rebound of cavity, defined by Bark [13] as "vortex rebound" and discussed in details by numerical illustration in [5].

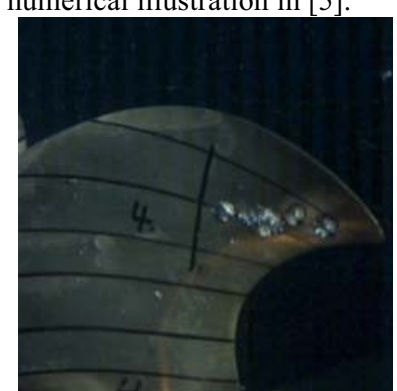

(a) frame 616

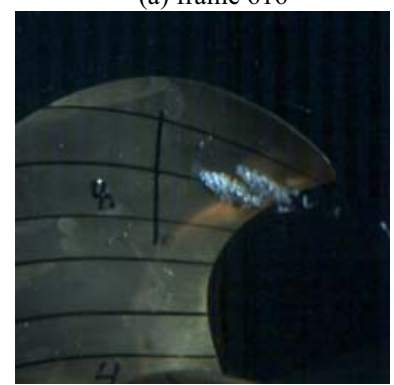

(c) frame 644

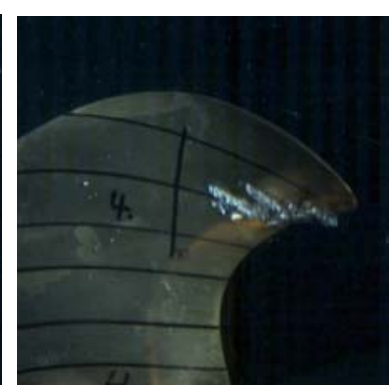

(b) frame 631

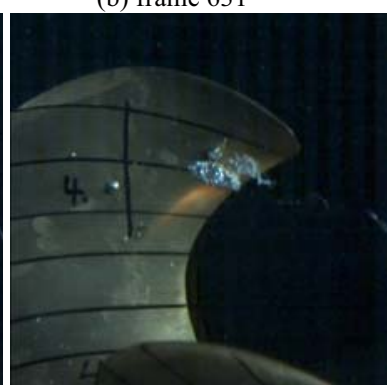

(d) frame 662
Figure 8: Side-frontal view of one blade passing by the upright position. There are at least traces of two vortices present on the blade that dominate the growth and affect the collapse of the sheet. $20000 \mathrm{f} / \mathrm{s}$.

For the cavitating flow, the potential flow method has failed to predict both the sheet on blade and the root cavitation. However this failure for this type of flow is not a surprise based on experience from previous results in [2].

\section{CONCLUDING REMARKS}

The performances in simulating dynamic cavitation on a marine propeller by Potential flow solver, RANS, and LES are respectively investigated both on quantitative and qualitative level against experimental data. Results indicate that although potential flow solver can predict fairly well the thrust and torque coefficient, and usually captures simple types of sheet cavitation, it is not suitable for neither prediction of more complex sheets, nor the prediction of root cavitation. RANS has partly captured the dynamic evolution of the sheet close to the tip region as well as the occurrence of the root cavitation, however it has mispredicted a leading edge sheet that is not present in the experiment. The missing of the vortical structure on blade limits also the use of RANS in analysis of some of the hydrodynamics that is crucial for understanding and controlling the cavitation and related noise and erosion. The LES computation shows the tendency in filling in this gap by capturing the correct location and dynamic behaviour of the vortical structure mentioned above. The grid resolution for the LES computation is known to be on the low side in this work in order to keep the same mesh to compare with the RANS computation, and therefore it is not expected to offer the smallscaled details actually needed about the near final collapse to make an assessment that can support or replace experiments. However with increased resolution in the region of interest, it is promising to use LES as a tool that can improve the understanding of the experimental phenomena and eventually offer guidance in propeller design.

\section{ACKNOWLEDGMENTS}

Financial support is provided by Rolls Royce AB through the University Technology Centre at the department of Shipping and Marine Technology, Chalmers. Experimental data is provided by Rolls-Royce Hydrodynamics Research Centre in Kristinehamn, Sweden.

\section{REFERENCES}

[1] Hämäläinen R., Lönnberg B., Arén P., Pettersson G., 2005, "Highest Comfort Class design for 'M/S Color Fantasy', the world's largest ever cruise-liner with car-deck", 1st int. ship noise \& vibration conf., London, UK.

[2] Proceedings of the Workshop on Cavitation and Propeller Performance Second International Symposium on Marine Propulsors - smp'11, 17 - 18 June 2011, Hamburg, Germany.

[3] Bensow R.E., Bark G., 2010, "Implicit LES prediction of the cavitating flow on a propeller," Journal of Fluids Engineering, 132(4), 041302 1-10.

[4] Hasuike N., Yamasaki S., and Ando J., 2011, ”Numerical and Experimental Investigation into Propulsion and Cavitation Performance of Marine Propeller," IV International Conference on Computational Methods in Marine Engineering, Lisbon, Portugal.

[5] Lu N.-X., Bensow R.E., Bark G., 2011, "Indicators of erosive cavitation in numerical simulations," The $7^{\text {th }}$ Internationl Workshop on Ship Hydrodynamics, Shanghai, China.

[6] Hirt C.W., Nichols, B.D., 1981, "Volume of fluid (VOF) method for the dynamics of free boundaries", Journal of Computational Physics 39 (1): 201-225.

[7] Kunz R., Boger F., Stinebring D.R., Chyczewski T.S., Lindau J.W., Gibeling H.J., Venkateswaran S., Govindan T.R., 2000, "A preconditioned Navier-Stokes method for two-phase flows with application to cavitation", Computers and Fluids, 29(8), pp. 849-875.

[8] Sauer J., Schnerr G.H., 2000, "Unsteady cavitating flow - a new cavitation model based on a modified front capturing method and bubble dynamics," Fluids Engineering Summer Conference, Proceeding of FEDSM'00.

[9] Lu N.-X., 2010, "Large Eddy Simulation of Cavitating Flow on Hydrofoils", Licentiate thesis, Chalmers University of Technology, Dept. Of Shipping and Marine Technology, Gothenburg, Sweden.

[10] Menter F. R., 1994, "Two-Equation Eddy-Viscosity Turbulence Models for Engineering Applications", AIAA Journal, 32 (8), 1598-1605.

[11] NASA 1976, "Vortex-lattice utilization," NASA SP-405, NASA-Langley, Washington.

[12] He, L., Chang, S-H., Kinnas, S., 2010., "MPUF-3A Version 3.0. User manual and documentation," University of Texas.

[13] Bark G., Grekula M., Lu N.-X., 2011, "Analysis of erosive cavitation by high speed video records". The 2nd International Conference on Advanced Model Measurement Technology for the EU Maritime Industry, Newcastle, UK. 